FRI0065

DHCR7 AND GENETIC POLYMORPHISMS IN THE ASSOCIATION BETWEEN VITAMIN D AND LIPID PROFILE IN RA: NEW PLAYERS IN CARDIOVASCULAR DISEASE?

J. Rodríguez-Carrio ${ }^{1,2,3}$, M. Alperi-López ${ }^{2,4}$, M. Naves-Díaz ${ }^{1,2}$, A. Dusso ${ }^{1,2}$, P. López ${ }^{2,3}$, F.J. Ballina-García ${ }^{2,4}$, J.B. Cannata-Andía ${ }^{1,2}$, A. Suárez ${ }^{2,3}$. ${ }^{1}$ Bone Mineral and Research Unit, Instituto Reina Sofía de Investigación Nefrológica, Redinren Isciii, Hospital Universitario Central de Asturias; ${ }^{2}$ Instituto de Investigación Sanitaria del Principado de Asturias (ISPA); ${ }^{3}$ Area of Immunology, University of Oviedo; ${ }^{4}$ Department of Rheumatology, Hospital Universitario Central de Asturias, Oviedo, Spain

Background: rheumatoid arthritis (RA) is associated with increased cardiovascu$\operatorname{lar}(\mathrm{CV})$ risk. Although vitamin $\mathrm{D}$ deficiency has been linked to $\mathrm{CV}$ disease in other conditions, studies in RA have yielded contradictory results and pointed to the involvement of additional factors. On the one hand, several genetic polymorphisms have been linked to vitamin D levels. Moreover, emerging evidence suggests a role for the enzyme DHCR7 in the crosstalk between cholesterol and vitamin $D$ synthesis.

Objectives: to evaluate the effect of genetic polymorphisms and DHCR7 levels in the interaction between vitamin $D$ and lipid profile in RA.

Methods: serum levels of vitamin D and DHCR7 were measured in 212 RA patients (2010 EULAR/ACR criteria) and 104 matched healthy controls (HC) by chemiluminescence and immunoassays, respectively. VDR rs2228750 and DHCR7 rs12785878 SNPs were studied by TaqMan Assays. The effect of vitamin $D$ deficiency on clinical features was assessed by linear regression analyses adjusting for age, gender, seasonality, vitamin D supplementation and DMARD usage.

Results: decreased vitamin $D$ was found in RA compared to $H C(p<0.001)$, vitamin $D$ deficiency $(<20 \mathrm{ng} / \mathrm{ml})$ being more prevalent in winter/spring $(58 / 151$, $38.4 \%)$ than in summer/autumn $(12 / 61,19.6 \% ; p=0.010)$. Vitamin $D$ levels were positively correlated to those of $\mathrm{HDL}(r=0.217, p<0.001)$, this association being restricted to patients with the rs2228750 AG/AA genotype $(r=0.287, p=0.003)$ but not in those with the $G G$ one $(r=0.082, p=0.531)$. Equivalent results were obtained for total/HDL-cholesterol ratio. Vitamin $D$ deficiency was associated with lower $\operatorname{HDL}(p=0.040)$, higher tender joint count $(p=0.003)$, swollen joint count $(p=0.005)$, DAS28 $(p=0.028)$ and HAQ $(p=0.030)$ in AG/AA-patients but not in those with the GG status (all $p>0.050$ ). Decreased DHCR7 levels were found between RA and $H C$ in winter/spring $(p=0.008)$ but not in summer/autumn $(p=0.116)$. RA patients with CV disease exhibited lower DHCR7 levels $(p=0.012)$ than their CV-free counterparts. Moreover, the associations among DHCR7, vitamin D and HDL levels followed a seasonal pattern: DHCR7 was correlated to vitamin D levels in summer/autumn $(r=0.441, p=0.004)$, where vitamin $D$ was associated with both HDL levels $(r=0.354, p=0.009)$ and total $/ \mathrm{HDL}$ ratio $(r=-0.393, p=0.003)$. A DHCR7 rs12785878-driven effect was observed for these associations. On the contrary, a weak correlation between $\mathrm{HDL}$ and vitamin $\mathrm{D}$ was found in winter/ spring $(r=0.215, p=0.014)$, where decreased DHCR7 was associated with age at onset $(r=-0.285, p=0.006)$ and $R F$ titre $(r=-0.326, p=0.013)$.

Conclusions: the effect of vitamin $D$ on the lipid profile is dependent on genetic polymorphisms. DHCR7 has a pivotal role in the interaction between vitamin D and lipid profile in RA. Seasonality and DHCR7-HDL axis may be novel mediators in the $\mathrm{CV}$ disease in RA.

Disclosure of Interest: None declared

DOI: 10.1136/annrheumdis-2018-eular.3199

\section{FRI0066 IMPACT OF SERUM ADIPOKINES IN EARLY STAGES OF RHEUMATOID ARTHRITIS}

J.A. Chaparro ${ }^{1,2}$, J.M. Bello-Gualtero ${ }^{1,2}$, W. Bautista-Molano $0^{2,3}$, L. Chila ${ }^{3}$, M. A. Cano ${ }^{3}$, R. Valle-Oñate ${ }^{1}$, P. Chalem ${ }^{4}$, C. Romero-Sánchez $z^{1,2,3} .{ }^{1}$ Rheumatology and Immunology, Hospital Militar Central; ${ }^{2}$ Clinical Immunology Group, Universidad Militar Nueva Granada; ${ }^{3}$ Unit of Oral Basic Investigation, Universidad El Bosque; ${ }^{4}$ Fundación Instituto de Reumatología Fernando Chalem, Bogotá D.C., Colombia

Background: Rheumatoid arthritis (RA) is a systemic chronic inflammatory disease. Adipose tissue is regarded as an active endocrine organ producing adipokines such as leptin and adiponectin, and some proinflammatory cytokines which have proinflammatory properties that account for chronic low-grade systemic inflammation

Objectives: To investigate the levels of adipokines in eRA and establish their association with the state of rheumatic condition

Methods: Cross sectional study was conducted. 51 patients with eRA according to the ACR/EULAR 2010 criteria and 51 healthy controls matched by age and gender, were included. A complete medical history was obtained. Adiponectin levels measured by Luminex technology, IL6 by chemiluminescence and Iptin by Enzyme-linked immunosorbent assay. Serum markers of RA such as rheumatoid factor, erythrocyte sedimentation rate(ESR), $C$ reactive protein(CRP), and anticitrullinated protein antibodies-APCA $\operatorname{lgG} / \lg$ A. Disease activity was evaluated by
DAS28CRP, DAS28ESR, CDAI, SDAI and RAPID3, the functional status using Multidimensional health assessment questionnaire(MDHAQ) and radiological status by Simple Erosion Narrowing Score(SENS). An association analysis was made to evaluate the relationship between adipokines levels and rheumatologic conditions using X2 test, Mann Whitney and logistic regression model was performed to confirm this associations. All the results were performed with a level of significance of $95 \%$

Results: In the eRA group, $80.39 \%$ were women, the mean age was 48.54 \pm 10.93 years. $37.25 \%$ had overweight and $13.72 \%$ had obesity Among the controls, $80.39 \%$ were women, with the mean age of $48.55 \pm 11.07$ years. $27.45 \%$ had overweight and $5.88 \%$ had obesity.45,09\% moderate activity and $17.64 \%$ high activity by DAS28ESR. High leptin levels were found in $64.7 \%$ vs $23.5 \%$ in healthy controls $(p=0,001)$. High adiponectin levels was found associated to $B M l<25$ $(p=0.017)$ and high leptin level to $B M l>25(p=0.038)$ and disease activity by RAPID3( $p=0.039)$. The presence of joint space narrowing in hands was associated with low adiponectin levels $(p=0.044)$. The association of leptin levels $>1.67$ $\mathrm{ng} / \mathrm{mL}$ with the diagnosis of RA was maintained in the model adjusted for the pres ence of $P$ gingivalis, DAS28-ESR, CRP $>3 \mathrm{mg} / \mathrm{L}$, economic status and $\mathrm{BMI}>30$, $\mathrm{OR}=2.7995 \% \mathrm{Cl}(1.54-5.07)$. The presence of high leptin and IL6 levels and low level adiponectin at the same time were more frequently found in the female gen$\operatorname{der}(p=0.047)$, primary schooling $(p=0.050), B M l>30(p=0.023)$, patients with radiological damage SENS $>3(p=0.047)$ and were found associated at the same time to $\mathrm{MDHAQ}>3, \mathrm{BMI}>25$ and RAPID3 >12 (OR 5.03 95\% Cl 1.05-24) adjusted to clinical variables

Conclusions: The role of adipokines as the leptin could be considered to be the bridge between immune tolerance, metabolic function and autoimmunity and sug gests the possibility to use circulating levels of adipokines as potential biomarkers of disease activity and functional status in early stage of rheumatoid arthritis

\section{REFERENCE:}

[1] Šenolt L. Expert Rev Clin Immunol 2017;13:1-3.

Disclosure of Interest: None declared

DOI: 10.1136/annrheumdis-2018-eular.3168

\section{FRI0067 \\ TRENDS IN THE INCIDENCE OF FELTY'S SYNDROME IN PATIENTS WITH RHEUMATOID ARTHRITIS IN SPAIN: AN OBSERVATIONAL COHORT STUDY OF HOSPITAL DISCHARGES FROM 1999 TO 2015 (TREND-AR STUDY)}

J.L. Morell ${ }^{1}$, R. Mazzucchelli ${ }^{2}$, E. Perez Fernandez ${ }^{3}$, J. Quiros ${ }^{4}$, C. Macia Villa ${ }^{5}$ N. Crespi ${ }^{6}$, M. Peña ${ }^{7}$, C. Barbadillo ${ }^{8}$, M. Espinosa ${ }^{8}$, H. Godoy ${ }^{8}$, M. FernandezPrada $^{9}$, M. Galindo ${ }^{10}$, A. Garcia Vadillo ${ }^{11}$, O. Guzon Illescas ${ }^{4}$, A. Herranz ${ }^{12}$, C. Martinez Prada ${ }^{13}$, C. Morado Qiñoa ${ }^{13}$, V. Villaverde ${ }^{14}$, on behalf of TREND-AR. ${ }^{1}$ Rheumatology, Hospital Universitario Ramón y Cajal; ${ }^{2}$ Rheumatology, Hospital Universitario Fundación Alcorcón; ${ }^{3}$ Rheumatology, Hospital Universitario Fundacion Alcorcon; ${ }^{4}$ Rheumatology, Hospital Universitario Fundación Alcorcon; ${ }^{5}$ Rheumatology, Hospital Universitario Severo Ochoa; ${ }^{6}$ C.S La Rivota; ${ }^{7}$ Rhaumatology, Hospital Universitario Fundación Alcorcon; ${ }^{8}$ Rheumatology, Hospital Universitario Puerta de Hierro, Madrid; ${ }^{9}$ Rheumatology, Hospital Universitario de Guadalajara, Guadalajara; ${ }^{10}$ Rheumatology, Hospital Universitario doce de octubre; ${ }^{11}$ Rheumatology, Hospital Universitario La Princesa;

${ }^{12}$ Rheumatology, Hospital del Henares; ${ }^{13}$ Rheumatology, Hospital Clinico San Carlos; ${ }^{14}$ Rheumatology, Hospital Universitario de Mostoles, Madrid, Spain

Background: Felty's syndrome (FS) is an unfrequent entity realted to Rheumatoid Arthritis (RA) but it's unknown the trend of FS in Spain.

Objectives: To analyse the incidence and trend of hospital admissions for FS in RA patients in Spain from 1999 to 2015.

Methods: We performed an observational retrospective population study analysing the spanish administrative database that includes a Minimun Basic Data Set (MBDS) of hospital admissions of RA patients from 1999 to 2015. We selected the MBDSs for FS. Cases were identified by the presence in primary and secondary diagnosis of its ICD9 code. The population at risk was estimated with an estimated prevalence of RA of $0,5 \%(0,8 \%$ women and $0,2 \%$ men). Crude and adjusted rates were calculated, and the trend was analysed using the Generalised Linear Model (GLM) with the year as the analysis variable. SPSS version 20 (SPSS Inc, Chicago, IL) was used.

Results: 338.343 RA hospital admissions were detected, being $802(0,2 \%)$ due to FS, $455(56,7 \%)$ women and $347(43,3 \%)$ men. Mean age was 67,94 (SD 13,76). There were $61(7,6 \%)$ deaths. Age-adjusted rate during the period was $42,19 / 10^{5}$ inhab. per year (19,76 women and 64,61 men). FS age-adjusted rate decreased from $25,90 / 10^{5}$ inhab. per year in 1999 to 17,20 in 2015 , both women (12,85 in 1999 to 11,75 in 2015) and men (80,15 in 1999 to 38,75 in 2015). An annual decrease in the FS rate of $0,5 \%$ is estimated (RRI 0,995; CI 95\% 0,9761,014). 
Conclusions: In Spain FS hospital admissions in patients with RA decreased between 1999-2015 with an estimation of $0,5 \%$ annual reduction not statistically significative.

Disclosure of Interest: None declared

DOI: 10.1136/annrheumdis-2018-eular.4755

\section{FRI0068 ULTRASONOGRAPHIC SUBCLINICAL CARDIOVASCULAR FINDINGS IN HISPANIC RA PATIENTS: A CASE-CONTROL STUDY}

J.R. Azpiri-López ${ }^{1}$, D.A. Galarza-Delgado ${ }^{2}$, I.J. Colunga-Pedraza², A.H. GuillénLozoya ${ }^{2}$, J.A. Dávila-Jiménez ${ }^{2}$, E.E. Abundis-Márquez ${ }^{2} .{ }^{1}$ Cardiology,

${ }^{2}$ Rheumatology, Hospital Universitario "Dr José E. Gonzalez", UANL, Monterrey, Mexico

Background: Patients with RA have a 1.5-2.0 fold increased risk of developing coronary artery disease (CAD) compared with the general population. Patients with RA are typically managed by several physicians, and coordination of care may be suboptimal. ${ }^{1}$ The leading cause of death in RA-patients is atherosclerotic cardiovascular disease (ASCVD). ${ }^{2}$ Carotid artery and heart evaluation by ultrasound is a useful tool for detection of cardiovascular conditions.

Objectives: To compare the presence of subclinical cardiovascular conditions among Mexican-mestizo RA patients and matched controls.

Methods: Design: Observational, cross-sectional, case-control study. Patients of 40 to 75 years old who fulfilled the 2010 ACR/EULAR and/or the 1987 ACR classification criteria for RA were consecutively enrolled. Patients with previous ASCVD or any other rheumatic disease were excluded. Two board-certified radiologist performed and assessed all carotid ultrasounds (cUS) and two board-certified cardiologist performed and assessed transthoracic echocardiography (TTE) according to guidelines of the American Society of Echocardiography. ASE, 2016 Results: The RA patients showed higher prevalence of cardiovascular risk conditions with respect to control population, such as concentric remodelling/hypertrophy, diastolic dysfunction, valve regurgitation and Carotid intima media thickness (CIMT) (See table 1).

Abstract FRI0068 - Table 1. Ecographic cardiovascular patological conditions among RA patients.

\begin{tabular}{|c|c|c|c|c|}
\hline Ecographic findings & $\underset{n=50(\%)}{R A}$ & $\begin{array}{c}\text { Controls } \\
n=50(\%)\end{array}$ & $P$ & O.R (C.I. 95\%) \\
\hline Valve regurgitation $n \%$ & $42(84)$ & $32(46)$ & 0.023 & $2.95(1.14-7.64)$ \\
\hline Aortic, $n(\%)$ & $4(8)$ & $3(6)$ & $0.999 *$ & $1.36(0.28-6.42)$ \\
\hline Tricuspid, n & $41(82)$ & $29(58)$ & 0.009 & $3.29(1.32-8.23)$ \\
\hline Mild, $n(\%)$ & $37(74)$ & $29(58)$ & 0.091 & $2.06(0.88-4.80)$ \\
\hline Moderate, $n \%$ & $4(8)$ & $0(0)$ & $0.126 *$ & $0.92(0.84-0.99)$ \\
\hline Mitral, $n(\%)$ & $28(56)$ & $6(12)$ & 0.0001 & $9.30(3.36-25.87)$ \\
\hline Pulmonar, n $\%$ & $8(16)$ & $2(4)$ & 0.046 & $4.57(0.91-22.73)$ \\
\hline Diastolic disfunction n $n \%$ & $34(68)$ & $16(32)$ & 0.003 & $3.46(1.52-7.90)$ \\
\hline LVCR n $\% \%$ & $13(26)$ & $6(12)$ & 0.074 & $2.50(0.89-7.44)$ \\
\hline LVH, $n \%$ & $5(10)$ & l(2) & 0.092 & $5.40(0.61-48.39)$ \\
\hline LVCRLVH, $n \%$ & $18(36)$ & $8(16)$ & 0.017 & $3.09(1.19-7.99)$ \\
\hline Mass index $(g / m 2)$, mean $=S D$ & $66.75 \pm 17.40$ & $73.11 \pm 22.49$ & 0.132 & $1.01(-14.67-1.93)$ \\
\hline$L V E F \%$, mean $=S D$ & $60.14 \pm 5.12$ & $63.42 \pm 5.67$ & 0.004 & $1.12(-5.49-1.06)$ \\
\hline GLS $(\%$, mean $=S D$ & $-20.73 \pm 2.90$ & $-21.03 \pm 2.48$ & 0.639 & $0.96(-0.96-1.56)$ \\
\hline TAPSE (mm), mean $\pm S D$ & $23.01 \pm 4.41$ & $24.06=3.12$ & 0.193 & $1.05(-2.6-0.54)$ \\
\hline CAWH $n(\%)$ & $21(42)$ & $10(20)$ & 0.017 & $2.80(1.18-7.06)$ \\
\hline Carotid plaque, $n \%$ & II (22) & $10(20)$ & 0.806 & $1.12(0.43-2.95)$ \\
\hline
\end{tabular}

$\mathrm{P}$ (P value) O.R. (Odds ratio) C.I. (Confidence interval) LVCR (Left ventricle concentric remodelation) LVH (Left Ventricle hypertrophy) LVEF (Left ventricle ejection fraction GLS (Global longitudinal strain) TAPSE (Tricuspid annular plane systolic excursion) CAWH (Carotid artery wall hypertrophy) SD (Standard deviation) * (Yates continuity correction)

Conclusions: This study reinforces the usefulness of cUS and TTE as a tool in the evaluation of cardiovascular conditions in RA patients. A systematic search of extra-articular and cardiovascular comorbidities should be mandatory in all RA patients.

\section{REFERENCES:}

[1] Crowson CS, Liao KP, Davis JM, Solomon DH, Matteson EL, Knutson KL, Gabriel SE. Rheumatoid arthritis and cardiovascular disease. American heart journal 2013;166(4):622-628

[2] Avina-Zubieta JA, Thomas J, Sadatsafavi M, Lehman AJ, Lacaille D. Risk of incident cardiovascular events in patients with rheumatoid arthritis: a meta-analysis of observational studies. Annals of the rheumatic diseases 2012;71(9):1524-9.

Acknowledgements: None

Disclosure of Interest: None declared

DOI: 10.1136/annrheumdis-2018-eular.7112

\section{FRI0069 \\ DIFFERENCE OF CLINICAL IMPLICATION REGARDING THE NUMBERS OF AUTOANTIBODIES AT PRESENTATION IN KOREAN PATIENTS WITH EARLY RHEUMATOID ARTHRITIS}

J.Y.Pyo ${ }^{1}$, C.-H. Mun ${ }^{2}$, S.M. Jung ${ }^{2}$, S.W. Lee ${ }^{2}$, J. Song ${ }^{2}$, S.K. Lee ${ }^{2}$, Y.B. Park ${ }^{2}$ ${ }^{1}$ Department of Internal Medicine, Yonsei University Wonju College of Medicine, Wonju; ${ }^{2}$ Department of Internal Medicine, Yonsei University College of Medicine Seoul, Korea, Republic Of

Background: Epitope spreading occurs prior to the clinical diagnosis of rheumatoid arthritis (RA), the number of peptide recognised and its titer increases remarkably as becoming close to the clinical diagnosis. However, the effect of multiple autoantibodies on the clinical outcome is not well known.

Objectives: This study is to investigate the association between the number of autoantibodies at presentation and the clinical aspects in Korean patients with early RA.

Methods: The number of baseline autoantibody (rheumatoid factor, anticitrullinated peptide antibodies (ACPA), and anti-carbamylated protein antibodies (antiCarP)) was analysed in the Korean Intensive Management of Early Rheumatoid Arthritis (KIMERA) cohort. All patients were disease-modifying antirheumatic drug (DMARD)-naïve RA patients with symptom duration less than 1 year. They were intensively treated by adjusting medications every 4 weeks, and treated to target as DAS28 of $<2.6$. Patients were classified regarding the number of autoantibod ies and clinical characteristics were compared between the groups.

Results: A total of 128 patients were included, seronegative patients showed higher baseline physician global VAS ( 7.3 vs 4.7 vs 4.6 vs $4.4, p=0.005)$ and DAS28-ESR (7.1 vs 4.5 vs 5.0 vs $4.7, p=0.003$ ) than patients with 1,2 , and 3 autoantibodies. Also seronegative patients showed a trend to have higher baseline $\mathrm{HAQ}$ score and pain VAS. After intensive treatment, there were no differences in remission rate and DAS28-ESR at 12,24 , and 36 months.

Abstract FRI0069 - Table 1. Disease characteristics regarding number of autoantibodies

\begin{tabular}{lccccc}
\hline Variables & $\mathbf{0}(\mathbf{n}=\mathbf{5})$ & $\mathbf{1}(\mathbf{n}=\mathbf{1 3})$ & $\mathbf{2}(\mathbf{n}=\mathbf{5 8})$ & $\mathbf{3}(\mathbf{n}=\mathbf{5 2})$ & $\mathbf{p}$-value \\
\hline Age, years & $58.2 \pm 16.2$ & $46.4 \pm 12.4$ & $52.8 \pm 12.9$ & $48.2 \pm 13.5$ & 0.104 \\
Female sex, N (\%) & $5(100 \%)$ & $7(53.8 \%)$ & $44(75.9 \%)$ & $44(84.6 \%)$ & 0.062 \\
Symptom duration, days & $7.0 \pm 4.8$ & $10.2 \pm 12.1$ & $10.5 \pm 16.0$ & $8.1 \pm 7.5$ & 0.736 \\
BMI (kg/m $\left.{ }^{2}\right)$ & $24.1 \pm 4.2$ & $21.7 \pm 1.9$ & $23.0 \pm 2.9$ & $23.0 \pm 3.1$ & 0.508 \\
ESR (mm/hour) & $59.0 \pm 15.8$ & $44.4 \pm 17.6$ & $56.8 \pm 32.0$ & $57.9 \pm 32.2$ & 0.543 \\
CRP (mg/L) & $20.9 \pm 17.3$ & $13.6 \pm 26.9$ & $19.0 \pm 29.4$ & $12.9 \pm 15.7$ & 0.559 \\
HAQ score & $1.4 \pm 0.5$ & $0.6 \pm 0.6$ & $0.7 \pm 0.6$ & $0.7 \pm 0.6$ & 0.092 \\
Pain VAS & $7.6 \pm 0.9$ & $4.0 \pm 2.0$ & $4.5 \pm 2.7$ & $4.4 \pm 2.9$ & 0.073 \\
Patient global VAS & $7.3 \pm 1.7$ & $4.7 \pm 2.2$ & $4.6 \pm 2.5$ & $4.4 \pm 2.7$ & 0.127 \\
Physician global VAS & $7.0 \pm 0.9$ & $4.9 \pm 1.1$ & $5.5 \pm 1.2$ & $4.5 \pm 1.6$ & 0.005 \\
DAS28-ESR & $7.1 \pm 0.6$ & $4.5 \pm 1.2$ & $5.0 \pm 1.4$ & $4.7 \pm 1.4$ & 0.003 \\
\hline
\end{tabular}

Values are expressed as means \pm standard deviation or $\mathrm{N}(\%)$. BMI, body mass index; ESR, erythrocyte sedimentation rate; $C R P, C$-reactive protein; $H A Q$ : health assessment questionnaire; VAS, visual analogue scale; DAS, disease activity score

\section{Changes in DAS28 according to number of autoantibodies}

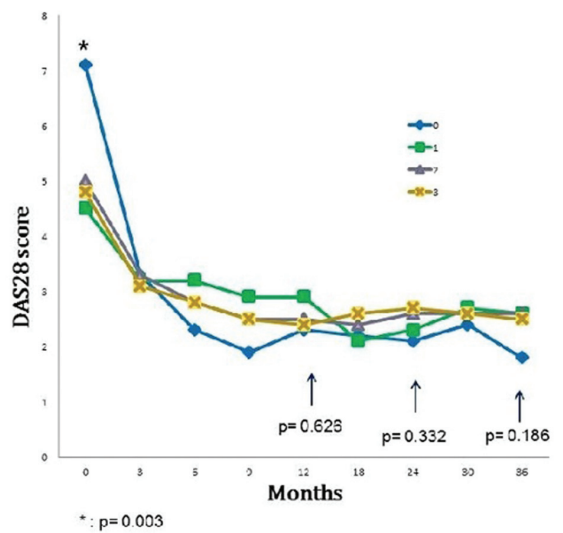

Conclusions: Seronegative patients showed higher levels of baseline inflammation than seropositive patients. However, they had favourable treatment response with rapid improvement which resulted in excellent clinical outcome.

Disclosure of Interest: None declared

DOI: 10.1136/annrheumdis-2018-eular.4354 